\title{
Recombinant Dense Granular Protein (GRA5) for Detection of Human Toxoplasmosis by Western Blot
}

\author{
Xiao Teng Ching, Yee Ling Lau, Mun Yik Fong, \\ Veeranoot Nissapatorn, and Hemah Andiappan \\ Department of Parasitology, Faculty of Medicine, University of Malaya, 50603 Kuala Lumpur, Malaysia \\ Correspondence should be addressed to Yee Ling Lau; lauyeeling@um.edu.my
}

Received 16 January 2014; Revised 14 May 2014; Accepted 15 May 2014; Published 29 May 2014

Academic Editor: Nongyao Sawangjaroen

Copyright (c) 2014 Xiao Teng Ching et al. This is an open access article distributed under the Creative Commons Attribution License, which permits unrestricted use, distribution, and reproduction in any medium, provided the original work is properly cited.

\begin{abstract}
Toxoplasma gondii infects all warm-blooded animals, including humans, causing serious public health problems and great economic loss for the food industry. Commonly used serological tests require costly and hazardous preparation of whole Toxoplasma lysate antigens from tachyzoites. Here, we have evaluated an alternative method for antigen production, which involved a prokaryotic expression system. Specifically, we expressed T. gondii dense granular protein-5 (GRA5) in Escherichia coli and isolated it by affinity purification. The serodiagnostic potential of the purified recombinant GRA5 (rGRA5) was tested through Western blot analysis against 212 human patient serum samples. We found that rGRA5 protein was $100 \%$ specific for analysis of toxoplasmosis-negative human sera. Also, rGRA5 was able to detect acute and chronic T. gondii infections (sensitivities of $46.8 \%$ and $61.2 \%$, resp.).
\end{abstract}

\section{Introduction}

Toxoplasmosis is a parasitic disease caused by Toxoplasma gondii (T. gondii) which belongs to phylum Apicomplexa [1]. It is an obligate intracellular protozoan parasite capable of infecting all warm-blooded domestic animals as well as human beings [2]. The infection is globally distributed affecting up to one-third of the world's human population [3]. Infection of $T$. gondii involves the transmission within and between hosts by zoites [4]. Three infectious stages of the parasite are tachyzoite, bradyzoite, and sporozoite [5]. Humans get infected with such disease through congenital transmission, consumption of raw or undercooked meat contaminated with T. gondii tissue cysts, or uptake of water contaminated with sporulated oocysts from the infected cat feces [6].

Toxoplasmosis in immunocompetent individuals is often asymptomatic [7] but can cause severe clinical outcome to immunocompromised patients leading to multisystem organ failure or even death [2]. Meanwhile, primary infection in pregnant women will likely transmit the parasite to the fetus vertically causing congenital toxoplasmosis and might eventually bring about miscarriage in pregnant women $[8,9]$.
Besides pregnant women, similar infection does occur in sheep and goats [10], giving rise to similar consequence. Abortions in these animals contribute to great economic loss in livestock industry. Therefore, it is crucial to conduct a rapid, highly accurate, and early diagnostic test for $T$. gondii infected patients/hosts for prevention or early treatment.

In general, there are few methods available for conducting laboratory diagnosis of toxoplasmosis including serologic assays (antibody detection), polymerase chain reaction (PCR; specific gene detection), histologic examination, and isolation of the parasite followed by inoculation into peritoneal cavities of mice (in vivo) or tissue cultures (in vitro) from biopsy tissue and blood/body fluids, respectively, of the infected patients [11]. However, the most commonly used diagnostic test would be the serological test which relies on the Toxoplasma lysate antigens (TLAs) from tachyzoites propagated in vivo or in vitro. There are several disadvantages pertaining to the usage of antigens originating from tachyzoites: high production cost, time consuming, inconstant quality, contamination with extraparasitic components, and exposure of the staff to the harmful living parasites [12]. To overcome this, recombinant DNA technology plays an important role in producing a larger quantity of recombinant 
antigenic proteins for serodiagnosis of $T$. gondii infection in a safer manner with lower production cost. Besides, recombinant tachyzoite proteins production either through prokaryotic or eukaryotic systems can reduce the variation of quality, enabling the development of a more specific and standardized serological assay.

Previous studies have reported the potential of various specific antigens of T. gondii such as the surface proteins [13, 14], microneme proteins [15], rhoptry proteins [16, 17], and dense granule proteins $[18,19]$ as seromarkers, either as single or multiantigen for detection of $T$. gondii-specific serum antibodies against acute (recently acquired) or/and chronic (distant past) Toxoplasma infection.

Dense granule (GRA) proteins are proteins with high immunogenicity [20]. They are found abundantly in both tachyzoites and bradyzoites [21] and make up most of the circulating antigens in the blood stream of an infected host which can be detected as early as a few hours postinfection (acute phase) [22]. GRA proteins were also found during the chronic stage of T. gondii infection [20]. As a result, the immunogenicity and prolonged expression of GRA proteins make them one of the promising candidates for recombinant protein production.

A total of 12 GRA proteins with the molecular weight ranging from 21 to $41 \mathrm{kDa}$ have been identified [4, 21, 23-25]. Diagnostic performance of GRA antigens such as GRA2, GRA6, GRA7, and GRA8 has been investigated via ELISA for discriminating acute from chronic Toxoplasma infections $[18,19,26-28]$. Recombinant GRA7 was also shown to detect acute $T$. gondii infection more strongly compared to chronic infection [29]. Meanwhile, in our previous study, sensitivity and specificity of recombinant GRA2 for serodiagnosis of Toxoplasma-infected patients' sera have also been evaluated through western blot which is capable of discriminating present from past infection [30]. More diagnostic candidates capable of detecting the early acquired phase of toxoplasmosis ought to be determined to improve the efficacy of serodiagnosis especially of pregnant women in order to reduce the risk of transplacental transmission.

Dense granule antigen 5 (GRA5) is a $21 \mathrm{kDa}$ hydrophobic protein consisting of a $\mathrm{N}$-terminal hydrophobic signal peptide and a hydrophobic transmembrane domain [31]. It was reported that GRA5 appears in both soluble and hydrophobic forms [32]. GRA5 is secreted into the parasitophorous vacuole (PV) by $T$. gondii as a soluble form during the host cell invasion [33] followed by transmembrane insertion into the parasitophorous vacuole membrane (PVM) with its $\mathrm{N}$ terminal projecting into the host cell cytoplasm, while Cterminus remains in the vacuole lumen [32]. A yeast twohybrid analysis with GRA5 [34] showed that this antigen binds to calcium modulating ligand (CALMG) for regulation of intracellular calcium concentration which helps to inhibit apoptosis [35] and further allows for long-term survival of $T$. gondii. Besides playing an important role in host cell invasion, maintenance of the PV, and long-term survival of the parasite, GRA5 was found to exist in all life stages of the parasite [36].

However, only limited studies were done on the evaluation of the potential of GRA5 as a diagnostic marker in Toxoplasma infection, thus making it a protein of interest to be studied in this research. Only one study has been conducted showing the suitability of the full-length recombinant GRA5 for use as a component of an antigen cocktail for the detection of anti-T. gondii IgG antibodies [37]. This research study was aimed at the production of recombinant GRA5 (designated rGRA5) antigen in bacteria and at evaluation of its immunogenic properties as a potential single-antigenic diagnostic candidate through western blot. At the same time, we will also find out if GRA5 can detect the early acute stage of human toxoplasmosis through this study.

\section{Materials and Methods}

2.1. Parasite. T. gondii tachyzoites (RH strain) were maintained by serial intraperitoneal passage in BALB/c mice and were harvested from the peritoneal fluids after 3 to 4 days of infection. The tachyzoites were washed and subsequently resuspended in sterile phosphate buffered saline (PBS) prior to usage.

2.2. Construction of Recombinant Plasmids. The T. gondii GRA5 gene sequence (corresponding to nucleotides 76-360), which encodes the GRA5 antigen, was obtained from Genbank (accession number: EU918733.1). DNA was extracted from tachyzoites of $T$. gondii (RH strain) and used as the template for PCR amplification of the GRA5 gene with forward ( $5^{\prime}$-GCGGAATTCGGTTCAACGCGTGAC- $\left.3^{\prime}\right)$ and reverse ( $5^{\prime}$-GACGAATTCCTCTTCCTCGGCAACTTC- $\left.3^{\prime}\right)$ primers, which introduced EcoRI restriction sites (underlined) to facilitate cloning. The PCR product was purified and cloned into the pRSET B prokaryotic expression vector (Invitrogen, USA) at the EcoRI site. The resulting recombinant GRA5-pRSET B construct permitted expression of an $\mathrm{N}$-terminally polyhistidine- (His-) tagged rGRA5 (amino acid residues 26-120), lacking its putative N-terminal signal sequence. Both the GRA5-pRSET B construct and the nonrecombinant pRSET $B$ plasmid were transformed into the prokaryotic expression host, Escherichia coli (E. coli) BL21(DE3)pLysS. The recombinant clones were screened and sequenced for verification purposes.

\subsection{Optimization of Heterologous Protein Expression in E. coli.} Optimal conditions for rGRA5 protein expression in E. coli were determined prior to scaling up the protein production protocol for further study. A single GRA5-pRSET Bcontaining colony was picked and inoculated into $5 \mathrm{~mL}$ of Luria-Bertani (LB) broth supplemented with ampicillin $(100 \mu \mathrm{g} / \mathrm{mL})$ and chloramphenicol $(34 \mu \mathrm{g} / \mathrm{mL})$. The culture was grown overnight at $37^{\circ} \mathrm{C}(200 \mathrm{rpm})$ and then diluted to a final volume of $10 \mathrm{~mL}$ with $\mathrm{LB}$ broth to yield an optical density of 0.1 at $600 \mathrm{~nm}\left(\mathrm{OD}_{600}\right)$. The culture was then grown at $37^{\circ} \mathrm{C}(\sim 250 \mathrm{rpm})$ until reaching an $\mathrm{OD}_{600}$ of 0.5 , at which point protein expression was induced by addition of different concentrations $(0.1,0.5$, and $1.0 \mathrm{mM})$ of isopropyl $\beta$-D-thiogalactopyranoside (IPTG; Invitrogen, USA) for various incubation periods $(0,2$, and $4 \mathrm{~h})$. The cells were harvested every hour by centrifugation at 5,000 $\times \mathrm{g}$ for $10 \mathrm{~min}$ 
before assessing protein expression using dodecyl sulphatepolyacrylamide gel electrophoresis (SDS-PAGE).

2.4. Expression and Purification of rGRA5. Large-scale protein production was achieved by inducing the culture with $1 \mathrm{mM}$ IPTG and incubating it for $2 \mathrm{~h}$ before harvesting by centrifugation. The Probond Purification System (Invitrogen, USA) and nitrilotriacetic acid-nickel (Ni-NTA; Qiagen, Germany) resin were then used to purify rGRA5, according to the manufacturers' instructions. Briefly, cell lysate was prepared under denaturing conditions prior to the purification steps. The cell pellet was resuspended in guanidine lysis buffer $(6 \mathrm{M}$ guanidine hydrochloride, $500 \mathrm{mM}$ sodium chloride, and $20 \mathrm{mM}$ sodium phosphate, $\mathrm{pH}$ 7.8) and rocked slowly for 5 to $10 \mathrm{~min}$ at room temperature to ensure thorough cell lysis, followed by sonication on ice with three 5-second pulses (high intensity). After sonication, the lysate was separated from cellular debris by centrifugation at $3,000 \times \mathrm{g}$ for $15 \mathrm{~min}$, added to a column with resin, and allowed to bind for $30 \mathrm{~min}$. Once the resin settled, the supernatant was aspirated, and the column was washed two times with each of the following: denaturing binding buffer ( $8 \mathrm{M}$ urea, $500 \mathrm{mM}$ sodium chloride, and $20 \mathrm{mM}$ sodium phosphate, $\mathrm{pH} 7.8$ ), denaturing wash buffer ( $8 \mathrm{M}$ urea, $500 \mathrm{mM}$ sodium chloride, and $20 \mathrm{mM}$ sodium phosphate, $\mathrm{pH} 6.0$ ), and denaturing wash buffer ( $8 \mathrm{M}$ urea, $500 \mathrm{mM}$ sodium chloride, and $20 \mathrm{mM}$ sodium phosphate, $\mathrm{pH}$ 5.3). The supernatant was aspirated after each washing step. After the last wash, the rGRA5 protein was eluted from the Ni-NTA resin with denaturing elution buffer $(8 \mathrm{M}$ urea, $500 \mathrm{mM}$ sodium chloride, and $20 \mathrm{mM}$ sodium phosphate, $\mathrm{pH}$ 4.0). E. coli carrying the empty pRSET B vector was used as a negative control for both expression and purification. The concentration of purified rGRA5 protein was measured with the Bradford Assay Kit (Bio-Rad, USA). The identity of the expressed and purified rGRA5 protein was confirmed by matrix-assisted laser desorption/ionization-time-of-flight (MALDI-TOF) mass spectrometry (MS).

2.5. In-Gel Tryptic Digestion of rGRA5. Affinity purified rGRA 5 was resolved by SDS-PAGE using $12 \%$ polyacrylamide gels, which were stained with Coomassie Brilliant Blue R-250 (Bio-Rad, USA) for $2 \mathrm{~h}$ and then incubated with destaining solution (7\% acetic acid, 5\% methanol) overnight at room temperature. The rGRA5 protein band was then excised from the Coomassie-stained gel (based on size) and further destained with $50 \mu \mathrm{L}$ of $50 \%$ acetonitrile (ACN) in $50 \mathrm{mM}$ ammonium bicarbonate $\left(\mathrm{NH}_{4} \mathrm{HCO}_{3}\right)$. This step was repeated several times (15-20 min washes, discarding the destaining solution after each wash) until the excised gel was completely destained. The rGRA5-containing gel plug was then incubated with $150 \mu \mathrm{L}$ of $10 \mathrm{mM}$ dithiothreitol (DTT) in $100 \mathrm{mM} \mathrm{NH} \mathrm{HCO}_{3}$ for $30 \mathrm{~min}$ at $60^{\circ} \mathrm{C}$. The gel was subsequently cooled to room temperature, the DTT solution was discarded, and the band was incubated with $150 \mu \mathrm{L}$ of $55 \mathrm{mM}$ iodoacetamide (IAA) in $100 \mathrm{mM} \mathrm{NH}_{4} \mathrm{HCO}_{3}$ in the dark for $20 \mathrm{~min}$. The gel plug was then washed four times with $50 \%$ $\mathrm{ACN}$ in $50 \mathrm{mM} \mathrm{NH} \mathrm{HCO}_{3}(500 \mu \mathrm{L}$ washes, 20 min each), dehydrated via incubation with $50 \mu \mathrm{L}$ of $100 \%$ ACN for
$15 \mathrm{~min}$, and subjected to speed vacuum for $15 \mathrm{~min}$ at ambient temperature to remove the $\mathrm{ACN}$. The gel plug was then incubated with $25 \mu \mathrm{L}$ of trypsin $(6 \mathrm{ng} / \mu \mathrm{L})$ in $50 \mathrm{mM} \mathrm{NH}_{4} \mathrm{HCO}_{3}$ at $37^{\circ} \mathrm{C}$. Following overnight digestion, $50 \mu \mathrm{L}$ of $50 \% \mathrm{ACN}$ was added to the gel plug, and it was incubated for $15 \mathrm{~min}$ in order to disintegrate the trypsin enzyme and extract protein from the gel plug. The resulting liquid (containing the digested protein) was transferred into a new tube (Tube A), and the gel plug, which remained in the old tube, was further incubated with $50 \mu \mathrm{L}$ of $100 \% \mathrm{ACN}$ for $15 \mathrm{~min}$. Subsequently, this liquid was also transferred to Tube A. The protein-containing solution in Tube A was then dried completely via speed vacuum. Prior to MALDI-TOF MS analysis, the protein sample was reconstituted in $10 \mu \mathrm{L}$ of $0.1 \%$ formic acid and desalted using a Zip-Tip (Millipore, USA). For this, the Zip-Tip membrane was wetted and equilibrated with $50 \%$ ACN and $0.1 \%$ formic acid, respectively. The protein sample was bound onto the Zip-Tip membrane, which was then washed with $0.1 \%$ formic acid. Finally, the protein was eluted with $0.1 \%$ formic acid in 50\% ACN and analyzed by MALDI-TOF MS.

2.6. MALDI-TOF MS Analysis. The Zip-Tip-eluted protein sample was mixed at a 1:1 ratio. The matrix was provided by UMCPR staff before spotting onto the MALDI plate. The analysis was carried out by University Malaya Center for Proteomics Research (UMCPR).

2.7. SDS-PAGE and Western Blot Analysis. Purified rGRA5 protein was resolved by SDS-PAGE on $12 \%$ polyacrylamide gels and transferred onto methanol-activated polyvinylidene difluoride (PVDF; Bio-Rad, USA) membranes, which were then cut into vertical strips. The membranes were incubated with blocking solution (5\% nonfat skim milk in Tris Buffered Saline (TBS)) for $2 \mathrm{~h}$ at room temperature with constant shaking and were subsequently probed with diluted human serum samples $(1: 200)$ for $2 \mathrm{~h}$. The membrane strips were washed and then incubated for $1 \mathrm{~h}$ with biotinylated goat antihuman IgM/IgG (KPL, USA; 1:2500) secondary antibody. Lastly, the membrane strips were washed and incubated with streptavidin-alkaline phosphatase (KPL, USA; $1: 2,500$ ) at room temperature for $1 \mathrm{~h}$ followed by detection using 5 bromo-4-chloro-3-indolyphosphate/nitro blue tetrazolium (BCIP/NBT; Sigma, USA).

2.8. Evaluation of Sensitivity and Specificity of rGRA5. Diagnostic sensitivity and specificity of rGRA5 protein were evaluated by western blot analysis using sera from both toxoplasmosis-diagnosed patients and toxoplasmosisnegative individuals. Toxoplasmosis cases were divided into three groups: (1) patients with early acute toxoplasmosis ( $n=44$; IgM positive, IgG negative); (2) patients with acute toxoplasmosis ( $n=47$; IgM positive, IgG positive); and (3) patients with chronic toxoplasmosis $(n=85$; IgM negative, IgG positive). A fourth group was comprised of toxoplasmosis-negative control patients $(n=24$; IgM negative, IgG negative). These human serum samples were grouped based on results obtained from Novalisa Toxoplasma gondii IgG and Toxoplasma gondii IgM enzyme-linked immunosorbent assay 
TABLE 1: Immunoreactivities (sensitivity and specificity) of the rGRA5 antigen to serum samples from toxoplasmosis-positive and toxoplasmosis-negative patients.

\begin{tabular}{|c|c|c|c|c|c|}
\hline \multirow{3}{*}{ Serum samples group } & \multirow{3}{*}{ Number of human serum samples } & \multicolumn{4}{|c|}{ Immunoreactivities } \\
\hline & & \multicolumn{2}{|c|}{ Positive } & \multicolumn{2}{|c|}{ Negative } \\
\hline & & Number & $\%$ & Number & $\%$ \\
\hline 1 (Early acute: IgG-ve, IgM+ve) & 44 & 0 & 0 & 44 & 100 \\
\hline 2 (Acute: IgG+ve, IgM+ve) & 47 & 22 & 46.8 & 25 & 53.2 \\
\hline 3 (Chronic: IgG+ve, IgM-ve) & 85 & 52 & 61.2 & 33 & 38.8 \\
\hline 4 (Toxoplasmosis-negative: IgG-ve, IgM-ve) & 24 & 0 & 0 & 24 & 100 \\
\hline Other infections & 12 & 1 & 8.3 & 11 & 91.7 \\
\hline Amoebiasis & 3 & 0 & 0 & 3 & 100 \\
\hline Cysticercosis & 3 & 0 & 0 & 3 & 100 \\
\hline Filariasis & 3 & 0 & 0 & 3 & 100 \\
\hline Toxocariasis & 3 & $1^{*}$ & 33.3 & 2 & 66.7 \\
\hline
\end{tabular}

* One out of three toxocariasis-positive sera samples reacted with the rGRA5 antigen. This particular toxocariasis-positive serum sample was shown to be IgG positive for toxoplasmosis based on the commercial kits.

(ELISA) kits (NovaTec, Germany). In addition, specificity of rGRA5 was determined using serum samples from patients diagnosed with amoebiasis (3 samples), cysticercosis (3 samples), filariasis (3 samples), and toxocariasis (3 samples). These sera had given positive results in serological tests for their respective infections. All serum samples were obtained from the Diagnostic Laboratory at the Department of Parasitology, University of Malaya. Sensitivity (number of true positives/[number of true positives + number of false negatives]) and specificity (number of true negatives/ [number of true negatives + number of false positives]) were calculated and tabulated in Table 1 .

\section{Results}

3.1. Cloning of the GRA5 Gene Fragment. We PCR-amplified a fragment of T. gondii GRA5 gene, which encoded amino acids 26-120 of the GRA5 protein (excluding the putative hydrophobic signal peptide). The resulting $\sim 285 \mathrm{bp}$ product was cloned into the pRSET B vector in order to permit prokaryotic expression of N-terminally His-tagged rGRA5, which could be purified using a nickel resin column. Sequence analysis confirmed that the insert within the GRA5pRSET B plasmid shared $100 \%$ identity with the published GRA5 gene.

3.2. Optimization of rGRA5 Expression in E. coli. Production of rGRA5 protein was optimized by altering various parameters, and expression levels were analyzed by SDS-PAGE as shown in Figure 1. Upon induction of rGRA5 expression from GRA5-pRSET B-containing E. coli, we observed a $20 \mathrm{kDa}$ band of increasing intensity, which was absent in the negative control (empty pRSET B). Expression of this protein increased up to two hours after induction and remained constant after four hours. Three different IPTG concentrations were tested, and $1.0 \mathrm{mM}$ was found to result in maximum rGRA5 expression. Taken together, these data suggested that optimal rGRA5 expression was achieved following induction

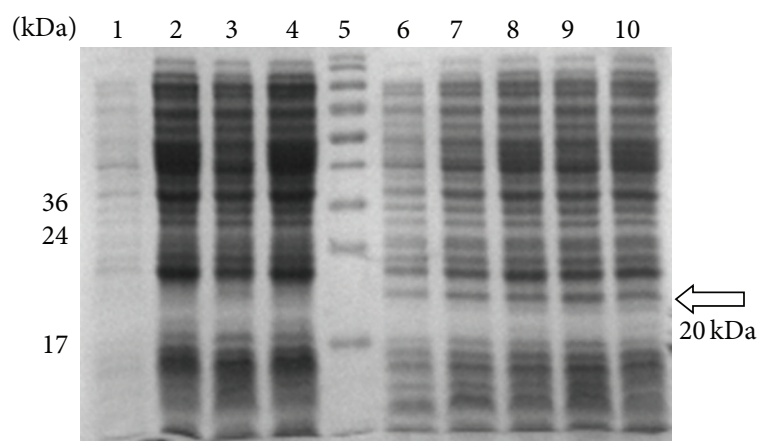

FIGURE 1: SDS-PAGE analysis on the optimized expression of rGRA5 protein in E. coli BL21 pLysS (DE3), Coomassie blue stained. Lane 5: prestained broad range protein marker. Lane 1, cell pellet fractions of pRSET B clone as negative control before induction $(0 \mathrm{hr})$. Lane 2: cell pellet fractions of pRSET B clone after induction with $0.5 \mathrm{mM}$ IPTG ( $4 \mathrm{hr}$ ). Lanes 3 to 4 : cell pellet fractions of pRSET B clone after induction with $1.0 \mathrm{mM} \operatorname{IPTG}(2,4 \mathrm{hr})$. Lane 6: cell pellet fractions of GRA5 clone before induction $(0 \mathrm{hr})$. Lanes 7 to 8: cell pellet fractions of GRA5 clone after induction with $0.5 \mathrm{mM}$ IPTG $(2,4 \mathrm{hr})$. Lanes 9 to 10: cell pellet fractions of GRA5 clone after induction with $1.0 \mathrm{mM}$ IPTG $(2,4 \mathrm{hr})$. The GRA5 protein band of interest was observed at molecular weight of $20 \mathrm{kDa}$ (arrow) compared to the negative control. The band intensity increased from 0 to $2 \mathrm{hr}$ after induction and remained constant at the 4 th $\mathrm{hr}$ with $1.0 \mathrm{mM}$ IPTG, the optimum condition for maximum expression of the protein.

with $1.0 \mathrm{mM}$ IPTG for 2 hours. The same conditions were applied to larger scale production of rGRA5.

3.3. Expression and Purification of rGRA5 Protein. Following optimization of rGRA5 expression in E. coli, a nickel resin column was used to purify the recombinant protein (Figure 2(a)), which could be detected by western blot analysis using serum from a Toxoplasma-infected patient (Figure 2(b)). This further suggested that the induced $20 \mathrm{kDa}$ band observed prior to purification corresponded to rGRA5 (Figure 1). 


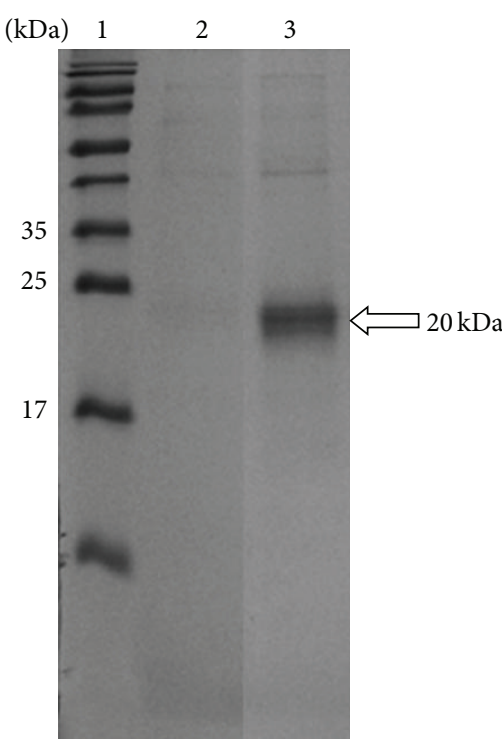

(a)

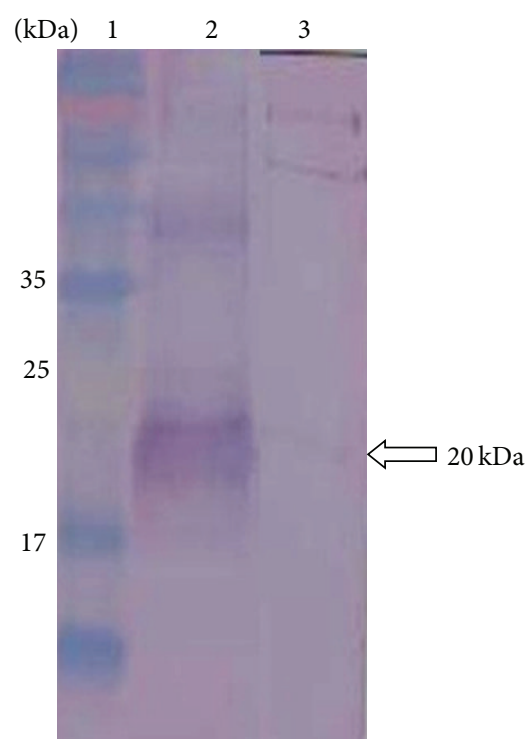

(b)

FIGURE 2: SDS-PAGE analysis on purified rGRA5 protein. (a) Coomassie blue stained. Lane 2: purified pRSET B. Lane 3: purified rGRA5 and (b) western blot probed with toxoplasmosis-infected patient's serum. Lane 2: purified rGRA5. Lane 3: purified pRSET B. Lane 1 (panel a and panel $b$ ) is the prestained broad range protein marker. The $20 \mathrm{kDa}$ purified rGRA5 was detected (arrow).

3.4. Confirmation of rGRA5 Protein. Next, we confirmed the identity of our expressed and purified recombinant protein by MALDI-TOF MS analysis. Indeed, the results indicated that the isolated protein was T. gondii GRA5.

\subsection{Western Blot Analysis of rGRA5 Protein with Human} Serum Samples. The purified rGRA5 protein was tested for its diagnostic sensitivity and specificity through western blot analysis with serum samples from toxoplasmosis-positive (Groups 1, 2, and 3) and toxoplasmosis-negative (Group 4) patients. In addition, specificity was tested using sera from patients infected with other parasites, including amoebiasis, cysticercosis, filariasis, and toxocariasis. We observed that the rGRA5 protein had sensitivities of $0 \%$ ( 0 out of 44 sera), $46.8 \%$ ( 22 out of 47 sera), and $61.2 \%$ (52 out of 85 sera) for early acute, acute, and chronic infections, respectively (Table 1). In contrast, 0 out of 24 control sera from the toxoplasmosis-negative patients reacted with rGRA5 (100\% specificity). In Figure 3, five example results are shown for each group (positive results for Groups 2 and 3; negative results for Group 4). Also, only 1 (toxocariasis) out of the 12 sera from patients infected with other parasites (data not shown) reacted with the rGRA5 protein (91.7\% specificity).

\section{Discussion}

A fragment of the T. gondii GRA5 gene was successfully cloned into a prokaryotic expression vector and transformed into E. coli. Full-length recombinant GRA5 protein (rGRA5) was subsequently expressed and purified, yielding a $20 \mathrm{kDa}$ protein. However, the predicted molecular weight of GRA5 is $16 \mathrm{kDa}$. While this discrepancy between the calculated and observed molecular weights can be partially explained by the presence of the His-tag in rGRA5, it is also possible that this difference stems from common features of GRA proteins, such as proline residue composition [4]. Even though we observed this size discrepancy, the identity of our purified protein was verified by immunoblotting with Toxoplasmainfected sera and MALDI-TOF MS analysis.

Identification of rGRA5 via MALDI-TOF MS involved careful processing, which allowed for reliable confirmation of the purified protein. Briefly, the rGRA5-containing band was excised from a stained SDS-PAGE gel, followed by an in-gel digestion protocol that included seven major steps: (1) destaining of the gel plug, (2) reduction of the protein, (3) alkylation of the protein, (4) dehydration, (5) tryptic digestion of the protein, (6) extraction of the digested protein, and (7) desalting of the digested protein using a Zip-Tip. Reduction and alkylation (aminocarboxymethylation) of the protein at cysteine residues with dithiothreitol (DTT) and iodoacetamide (IAA), respectively, were important for permanent disruption of disulfide linkages, enabling overnight trypsin digestion.

It was demonstrated that the expression of predicted immunodominant epitopes of GRA5 failed to show any immunoreactivity with a pool of T. gondii-positive human sera [13]. Therefore, full-length rGRA5 was constructed and produced in this study. Our evaluation of rGRA5 immunoreactivity revealed high specificities when testing sera from toxoplasmosis-negative patients or from those infected with other parasites (100.0\% and $91.7 \%$, resp.). In addition, our findings indicate the sensitivities of $46.8 \%$ and $61.2 \%$ when analyzing serum samples from patients with acute and chronic Toxoplasma infections, respectively. However, none of the serum samples from the early acute phase patients 

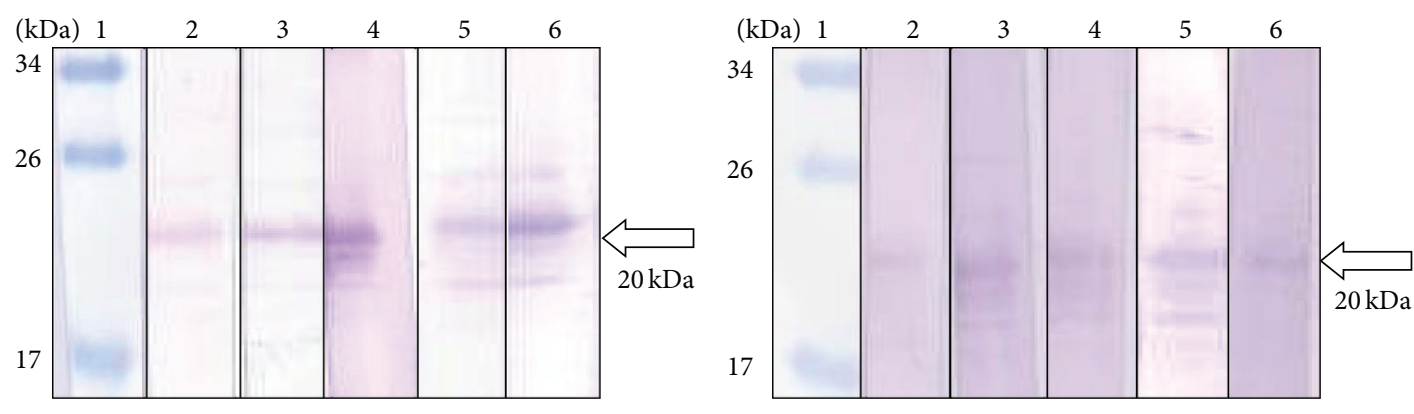

(a)

(b)

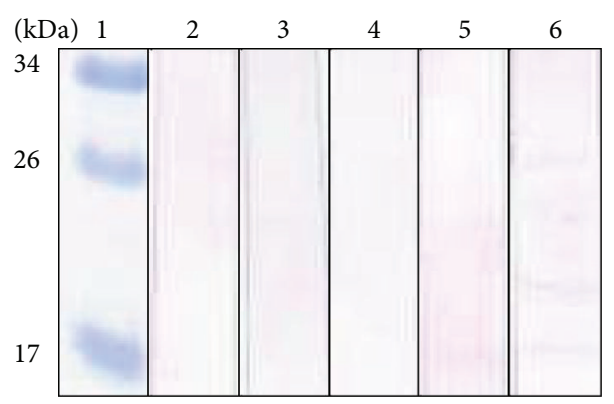

(c)

FIGURE 3: Western blots of purified rGRA5 protein with sera of toxoplasmosis and toxoplasmosis-negative patients. Lane 1 (panels a-c): the prestained broad range protein marker. Lanes 2 (a) to 6 (a): results of 5 sera from chronic-profile patients (Group 3: IgG +ve, IgM -ve). Lanes 2 (b) to 6 (b): results of 5 sera from acute-profile patients (Group 2: IgG +ve, IgM +ve). Lanes 2 (c) to 6 (c): results of 5 sera from toxoplasmosisnegative patients (Group 4: IgG -ve, IgM -ve). The $20 \mathrm{kDa}$ purified rGRA5 was detected by toxoplasmosis-positive sera (arrow).

reacted with the rGRA5 protein. In fact, data from the present study are in agreement with previous results obtained from analysis of rGRA5 antigen-mediated detection of IgG antibodies using ELISA [37]. Specificity of the aforementioned study was shown to be $100.0 \%$, whereas sensitivities of $63.0 \%$ and $75.0 \%$ were reported for sera from acute and chronic infections, respectively. Thus, it strongly suggested that rGRA5 yields a much higher reactivity towards IgG antibodies in sera from chronically infected patients compared to those with acute infection. Notably, this protein shows no sensitivity towards IgM antibodies found in sera from early acute stage patients. Our study involved the same expression host, BL21(DE3)pLysS, for the expression of full-length rGRA5 as the above mentioned study. In contrast, different expression vectors and evaluation techniques were used. Due to its higher specificity, western blot was chosen to evaluate rGRA5 protein in this study instead of the commonly used ELISA. Also, the chances of obtaining false-positive results via western blot are much lower compared to ELISA [38]. In fact, it has been reported that western blot analysis is superior to ELISA for screening sera samples because this technique gives more information, is less affected by sample degradation, produces results of high confidence with direct visualization of antibodies bound to specific diagnostic antigens, and offers improved determination of diagnostic antigen purity [39].

With regard to the future development of diagnostic tests for $T$. gondii, the western blot results obtained in this study should be reliable for predicting the efficacy of using rGRA5 antigen in immunochromatographic tests (ICT) due to similarities between the two assays (i.e., western blot and ICT are both immunoassays utilizing nitrocellulose membranes and direct visualization of results). Indeed, ICT is a better serological test for diagnosis of infections (including toxoplasmosis) compared to ELISA, which is commonly used due to its simplicity. However, ICT is a rapid test with high accuracy but lower cost compared to ELISA, which is time consuming and laborious [40]. In addition, ICT can be used in field conditions [40] especially for the diagnosis of farm animals.

Based on our results (Table 1), cross-reactivity was not observed in sera samples from patients infected with amoebiasis, cysticercosis, and filariasis. However, one out of three toxocariasis-positive sera samples reacted with the rGRA5 antigen. This particular toxocariasis-positive serum sample was shown to be IgG positive but IgM negative for toxoplasmosis based on findings from Novalisa Toxoplasma gondii IgG and IgM antibodies ELISA kits. This indicates that there was probably a coinfection of T. gondii and Toxocara spp. in this infected patient [41]. Although T. gondii (a protozoan) and Toxocara spp. (helminths) are two different parasites, they both can be acquired through soil ingestion. Therefore, the chances of coinfection between these two parasites are highly possible [41].

\section{Conclusions}

Our findings show that rGRA5 lacks sensitivity for detecting IgM antibodies and displays a much lower reactivity towards 
IgG antibodies in sera from patients with acute infection compared to those with chronic toxoplasmosis (46.8\% versus $61.2 \%)$. These data indicate that rGRA5 protein is unable to distinguish between current and past infections. Nevertheless, this protein can be combined with other T. gondii antigens (cocktails) in order to improve its sensitivity against toxoplasmosis-positive serum samples [37]. Last but not least, these findings should contribute to the future development of an ICT incorporating this antigen (either alone or in combination with other potential ESA) for diagnosis of T. gondii infection.

\section{Ethical Approval}

The authors declared that the experiments comply with the current laws of the country in which they were performed (Malaysia).

\section{Conflict of Interests}

The authors declare that there is no conflict of interests regarding the publication of this paper.

\section{Acknowledgments}

The authors thank the Diagnostic Laboratory (Para: SEAD), Department of Parasitology, University of Malaya, and University Malaya Medical Centre (UMMC) for providing the serum samples. They also thank the University Malaya Centre for Proteomics Research (UMCPR) for carrying out the MALDI-TOF MS analysis. This research project is supported by University of Malaya under the Postgraduate Research Grant (PS197-2009C) and High Impact Research Fund UMMOHE UM.C/HIR/MOHE/MED/16 from the Ministry of Higher Education in Malaysia.

\section{References}

[1] M. Igarashi, F. Kano, K. Tamekuni et al., "Toxoplasma gondii: evaluation of an intranasal vaccine using recombinant proteins against brain cyst formation in BALB/c mice," Experimental Parasitology, vol. 118, no. 3, pp. 386-392, 2008.

[2] A. B. Ismael, D. Sekkai, C. Collin, D. Bout, and M.-N. Mévélec, "MIC3 gene of Toxoplasma gondii is a novel potent vaccine candidate against toxoplasmosis," Infection and Immunity, vol. 71, no. 11, pp. 6222-6228, 2003.

[3] A. M. Tenter, A. R. Heckeroth, and L. M. Weiss, "Toxoplasma gondii: from animals to humans," International Journal for Parasitology, vol. 30, no. 12-13, pp. 1217-1258, 2000.

[4] C. Mercier, K. D. Z. Adjogble, W. Däubener, and M.-F. Delauw, "Dense granules: are they key organelles to help understand the parasitophorous vacuole of all apicomplexa parasites?" International Journal for Parasitology, vol. 35, no. 8, pp. 829-849, 2005.

[5] J. P. Dubey, D. S. Lindsay, and C. A. Speer, "Structures of Toxoplasma gondii tachyzoites, bradyzoites, and sporozoites and biology and development of tissue cysts," Clinical Microbiology Reviews, vol. 11, no. 2, pp. 267-299, 1998.
[6] J. P. Dubey, "Pathogenicity and infectivity of Toxoplasma gondii oocysts for rats," Journal of Parasitology, vol. 82, no. 6, pp. 951956, 1996.

[7] R. Chen, S.-H. Lu, Q.-B. Tong et al., "Protective effect of DNAmediated immunization with liposome-encapsulated GRA4 against infection of Toxoplasma gondii," Journal of Zhejiang University: Science B, vol. 10, no. 7, pp. 512-521, 2009.

[8] S. S. Gagne, “Toxoplasmosis," Primary Care Update for OB/ GYNS, vol. 8, no. 3, pp. 122-126, 2001.

[9] J. G. Montoya and O. Liesenfeld, "Toxoplasmosis," The Lancet, vol. 363, no. 9425, pp. 1965-1976, 2004.

[10] D. Buxton, "Protozoan infections (Toxoplasma gondii, Neospora caninum and Sarcocystis spp.) in sheep and goats: recent advances," Veterinary Research, vol. 29, no. 3-4, pp. 289-310, 1998.

[11] J. G. Montoya, "Laboratory diagnosis of Toxoplasma gondii infection and toxoplasmosis," Journal of Infectious Diseases, vol. 185, no. 1, supplement, pp. S73-S82, 2002.

[12] J. Gatkowska, E. Hiszczynska-Sawicka, J. Kur, L. Holec, and H. Dlugonska, "Toxoplasma gondii: an evaluation of diagnostic value of recombinant antigens in a murine model," Experimental Parasitology, vol. 114, no. 3, pp. 220-227, 2006.

[13] J. Dai, M. Jiang, Y. Wang, L. Qu, R. Gong, and J. Si, “Evaluation of a recombinant multiepitope peptide for serodiagnosis of Toxoplasma gondii infection," Clinical and Vaccine Immunology, vol. 19, no. 3, pp. 338-342, 2012.

[14] Y. L. Lau, G. Thiruvengadam, W. W. Lee, and M. Y. Fong, "Immunogenic characterization of the chimeric surface antigen 1 and 2 (SAG1/2) of Toxoplasma gondii expressed in the yeast Pichia pastoris," Parasitology Research, vol. 109, no. 3, pp. 871878, 2011.

[15] L. Holec, A. Gasior, A. Brillowska-Dabrowska, and J. Kur, “Toxoplasma gondii: enzyme-linked immunosorbent assay using different fragments of recombinant microneme protein 1 (MICl) for detection of immunoglobulin G antibodies," Experimental Parasitology, vol. 119, no. 1, pp. 1-6, 2008.

[16] L. Holec-Ģsior, J. Kur, and E. Hiszczyńska-Sawicka, "GRA2 and ROP1 recombinant antigens as potential markers for detection of Toxoplasma gondii-specific immunoglobulin G in humans with acute toxoplasmosis," Clinical and Vaccine Immunology, vol. 16, no. 4, pp. 510-514, 2009.

[17] P. van Gelder, F. Bosman, F. De Meuter, H. Van Heuverswyn, and P. Herion, "Serodiagnosis of toxoplasmosis by using a recombinant form of the 54-kilodalton rhoptry antigen expressed in Escherichia coli," Journal of Clinical Microbiology, vol. 31, no. 1, pp. 9-15, 1993.

[18] M. Golkar, K. Azadmanesh, G. Khalili et al., "Serodiagnosis of recently acquired Toxoplasma gondii infection in pregnant women using enzyme-linked immunosorbent assays with a recombinant dense granule GRA6 protein," Diagnostic Microbiology and Infectious Disease, vol. 61, no. 1, pp. 31-39, 2008.

[19] M. Golkar, S. Rafati, M. S. Abdel-Latif et al., "The dense granule protein GRA2, a new marker for the serodiagnosis of acute Toxoplasma infection: comparison of sera collected in both France and Iran from pregnant women," Diagnostic Microbiology and Infectious Disease, vol. 58, no. 4, pp. 419-426, 2007.

[20] C. Mercier, M. F. Cesbron-Delauw, and D. J. P. Ferguson, "Dense granules of the infectious stages of Toxoplasma gondii: their central role in the host/parasite relationship," in Toxoplasma: Molecular and Cellular Biology, D. Soldati and J. Ajioka, Eds., Horizon Scientific Press, 2007. 
[21] M.-F. Cesbron-Delauw, "Dense-granule organelles of Toxoplasma gondii: their role in the Host-Parasite relationship," Parasitology Today, vol. 10, no. 8, pp. 293-296, 1994.

[22] H. P. A. Hughes and F. van Knapen, "Characterisation of a secretory antigen from Toxoplasma gondii and its role in circulating antigen production," International Journal for Parasitology, vol. 12, no. 5, pp. 433-437, 1982.

[23] H.-J. Ahn, S. Kim, and H.-W. Nam, "Host cell binding of GRA10, a novel, constitutively secreted dense granular protein from Toxoplasma gondii," Biochemical and Biophysical Research Communications, vol. 331, no. 2, pp. 614-620, 2005.

[24] A. Michelin, A. Bittame, Y. Bordat et al., "GRA12, a Toxoplasma dense granule protein associated with the intravacuolar membranous nanotubular network," International Journal for Parasitology, vol. 39, no. 3, pp. 299-306, 2009.

[25] M. E. Rome, J. R. Beck, J. M. Turetzky, P. Webster, and P. J. Bradley, "Intervacuolar transport and unique topology of GRA14, a novel dense granule protein in Toxoplasma gondii," Infection and Immunity, vol. 76, no. 11, pp. 4865-4875, 2008.

[26] D. Jacobs, M. Vercammen, and E. Saman, "Evaluation of recombinant dense granule antigen 7 (GRA7) of Toxoplasma gondii for detection of immunoglobulin $\mathrm{G}$ antibodies and analysis of a major antigenic domain," Clinical and Diagnostic Laboratory Immunology, vol. 6, no. 1, pp. 24-29, 1999.

[27] S. Li, G. Maine, Y. Suzuki et al., "Serodiagnosis of recently acquired Toxoplasma gondii infection with a recombinant antigen," Journal of Clinical Microbiology, vol. 38, no. 1, pp. 179-184, 2000.

[28] A. Redlich and W. A. Müller, "Serodiagnosis of acute toxoplasmosis using a recombinant form; of the dense granule antigen GRA6 in an enzyme-linked immunosorbent assay," Parasitology Research, vol. 84, no. 9, pp. 700-706, 1998.

[29] G. Sadeghiani, M. Zare, J. Babaie et al., "Heterologous production of dense granule gra7 antigen of Toxoplasma gondii in Escherichia coli," Southeast Asian Journal of Tropical Medicine and Public Health, vol. 40, no. 4, pp. 692-700, 2009.

[30] X. T. Ching, Y. L. Lau, M. Y. Fong, and V. Nissapatorn, "Evaluation of Toxoplasma gondii-recombinant dense granular protein (GRA2) for serodiagnosis by western blot," Parasitology Research, vol. 112, no. 3, pp. 1229-1236, 2013.

[31] H.-W. Nam, "GRA proteins of Toxoplasma gondii: maintenance of host-parasite interactions across the parasitophorous vacuolar membrane," Korean Journal of Parasitology, vol. 47, supplement, pp. S29-S37, 2009.

[32] L. Lecordier, C. Mercier, L. David Sibley, and M.-F. CesbronDelauwz, "Transmembrane insertion of the Toxoplasma gondii GRA5 protein occurs after soluble secretion into the host cell," Molecular Biology of the Cell, vol. 10, no. 4, pp. 1277-1287, 1999.

[33] L. Lecordier, C. Mercier, G. Torpier et al., "Molecular structure of a Toxoplasma gondii dense granule antigen (GRA 5) associated with the parasitophorous vacuole membrane," Molecular and Biochemical Parasitology, vol. 59, no. 1, pp. 143-153, 1993.

[34] H.-J. Ahn, S. Kim, H.-E. Kim, and H.-W. Nam, "Interactions between secreted GRA proteins and host cell proteins across the paratitophorous vacuolar membrane in the parasitism of Toxoplasma gondii," The Korean Journal of Parasitology, vol. 44, no. 4, pp. 303-312, 2006.

[35] P. Feng, J. Park, B.-S. Lee, S.-H. Lee, R. J. Bram, and J. U. Jung, "Kaposi's sarcoma-associated herpesvirus mitochondrial K7 protein targets a cellular calcium-modulating cyclophilin ligand to modulate intracellular calcium concentration and inhibit apoptosis," Journal of Virology, vol. 76, no. 22, pp. 11491-11504, 2002.

[36] M. Tilley, M. E. Fichera, M. K. Jerome, D. S. Roos, and M. W. White, "Toxoplasma gondii sporozoites form a transient parasitophorous vacuole that is impermeable and contains only a subset of dense-granule proteins," Infection and Immunity, vol. 65, no. 11, pp. 4598-4605, 1997.

[37] L. Holec-Gasior and J. Kur, "Toxoplasma gondii: recombinant GRA5 antigen for detection of immunoglobulin G antibodies using enzyme-linked immunosorbent assay," Experimental Parasitology, vol. 124, no. 3, pp. 272-278, 2010.

[38] K. Nöckler, S. Reckinger, A. Broglia, A. Mayer-Scholl, and P. Bahn, "Evaluation of a Western Blot and ELISA for the detection of anti-Trichinella-IgG in pig sera," Veterinary Parasitology, vol. 163, no. 4, pp. 341-347, 2009.

[39] T. Anderson, A. DeJardin, D. K. Howe, J. P. Dubey, and M. L. Michalski, "Neospora caninum antibodies detected in Midwestern white-tailed deer (Odocoileus virginianus) by Western blot and ELISA," Veterinary Parasitology, vol. 145, no. 1-2, pp. 152$155,2007$.

[40] X. Huang, X. Xuan, H. Hirata et al., "Rapid immunochromatographic test using recombinant SAG2 for detection of antibodies against Toxoplasma gondii in cats," Journal of Clinical Microbiology, vol. 42, no. 1, pp. 351-353, 2004.

[41] J. L. Jones, D. Kruszon-Moran, K. Won, M. Wilson, and P. M. Schantz, "Toxoplasma gondii and Toxocara spp. co-infection," American Journal of Tropical Medicine and Hygiene, vol. 78, no. 1, pp. 35-39, 2008. 


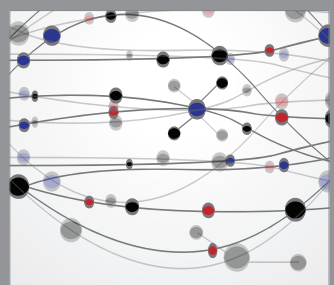

The Scientific World Journal
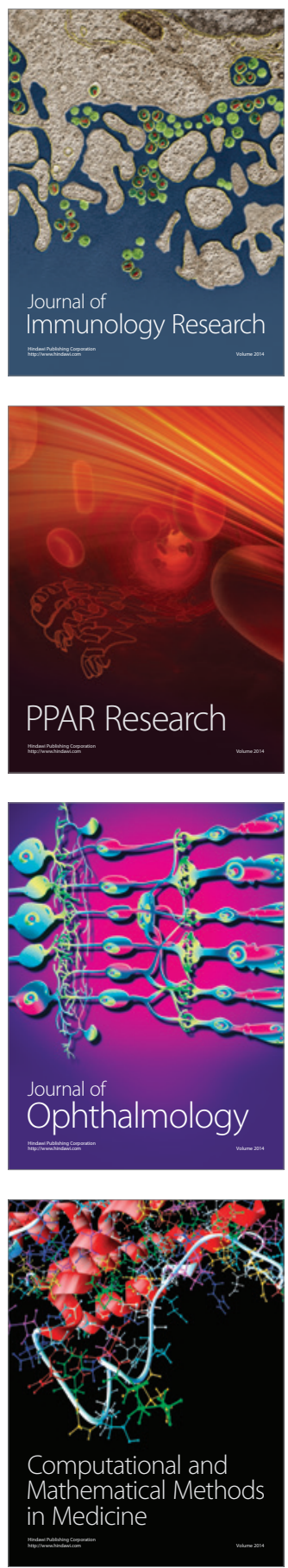

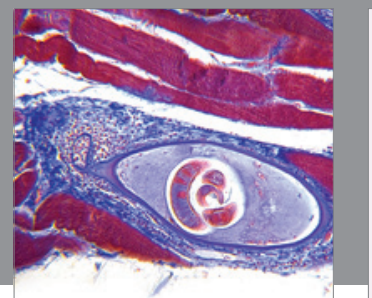

Gastroenterology

Research and Practice
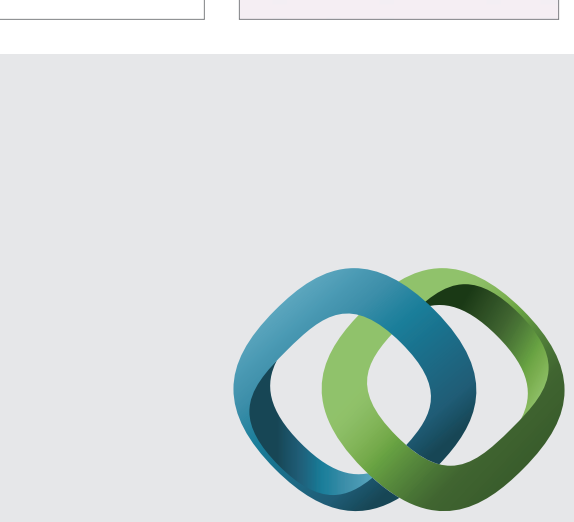

\section{Hindawi}

Submit your manuscripts at

http://www.hindawi.com
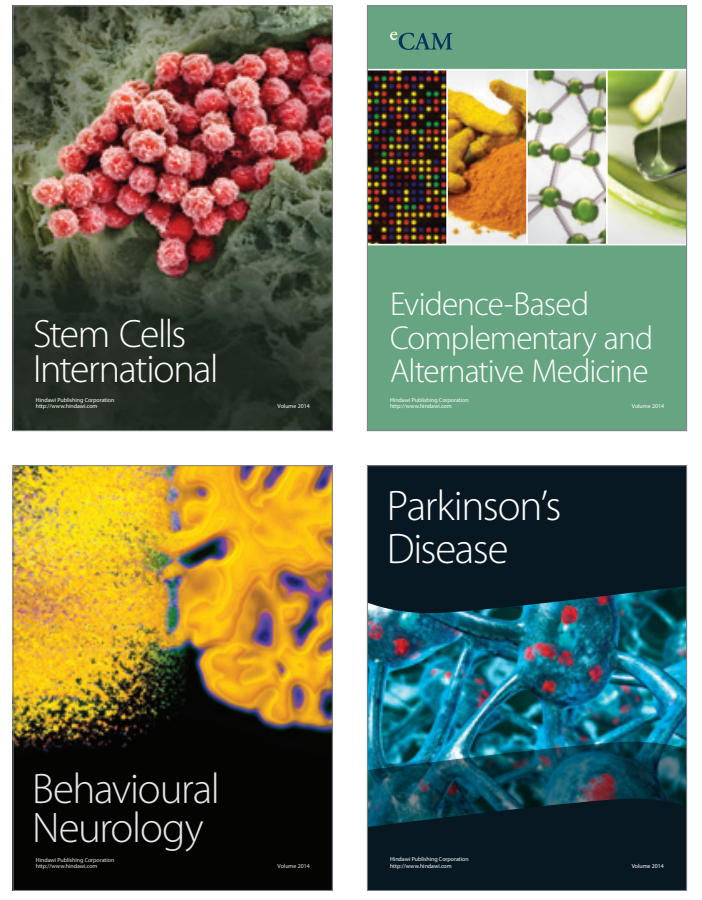
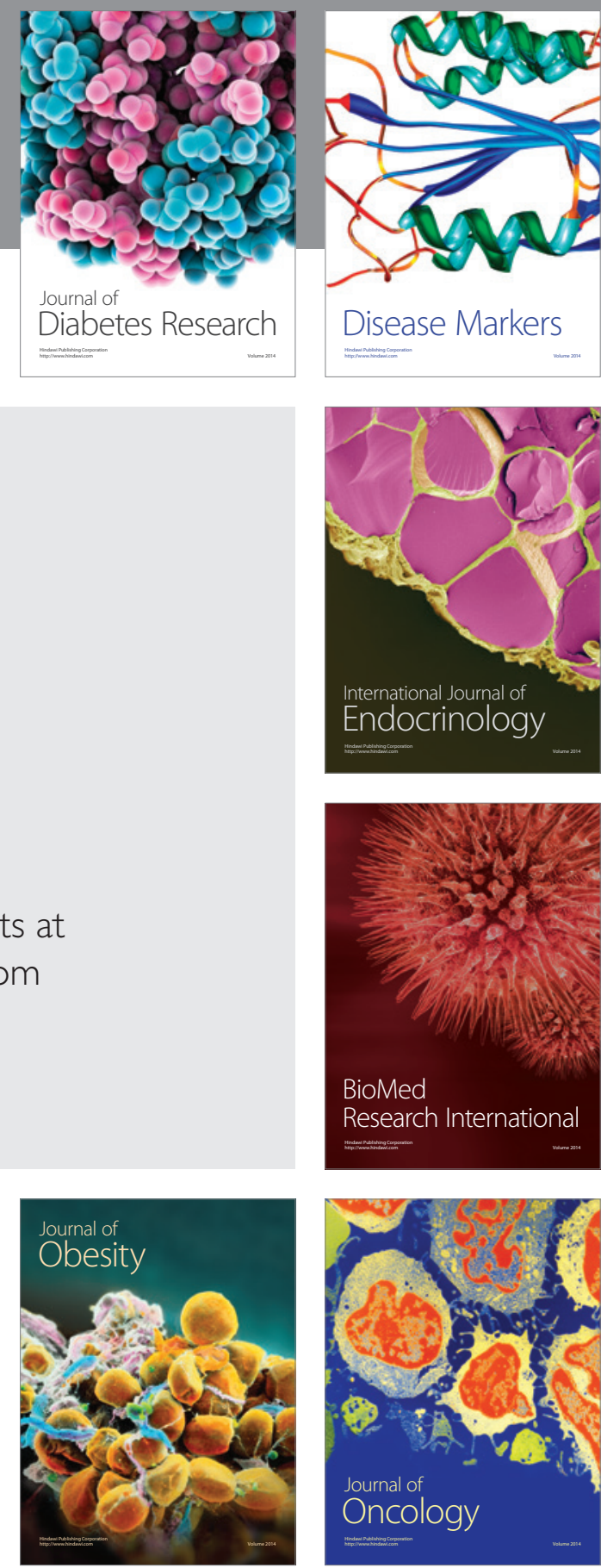

Disease Markers
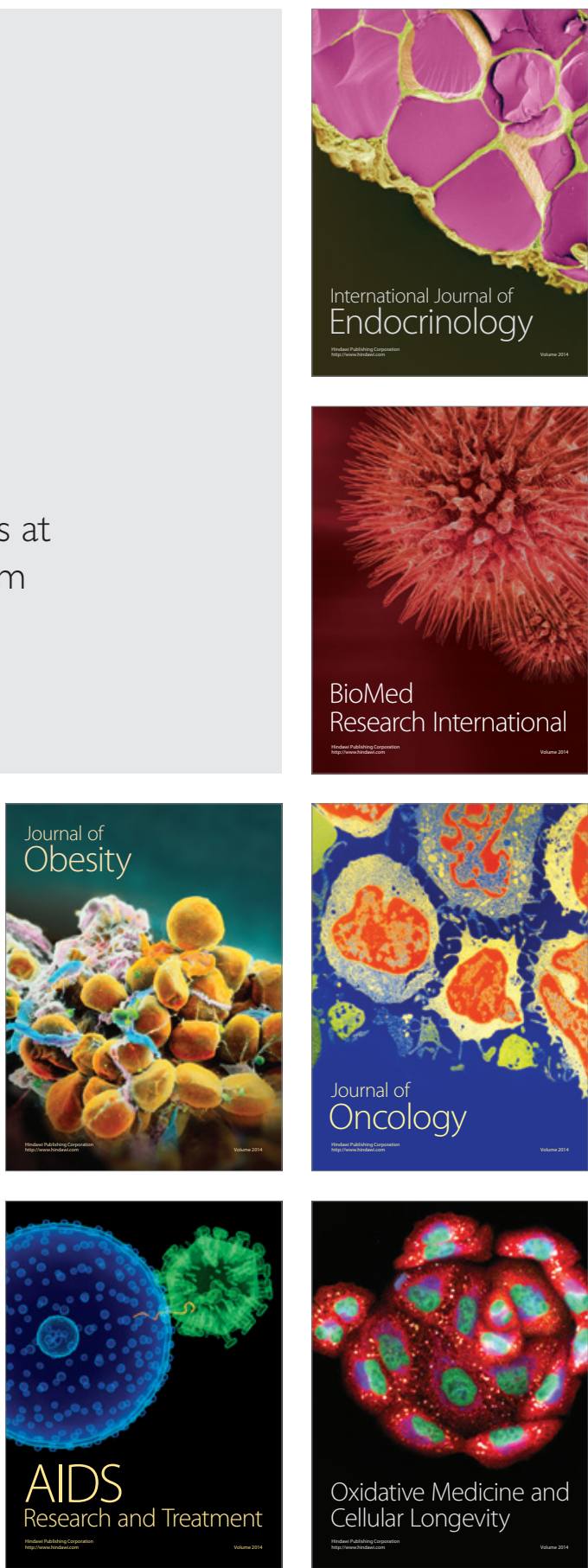